\title{
The Clinical Effectiveness of Ranibizumab Treat and Extend Regimen in nAMD: Systematic Review and Network Meta-Analysis
}

\author{
Andriy Danyliv (D) Julie Glanville · Rachael McCool · Alberto Ferreira • \\ Adrian Skelly $\cdot$ Ruth Pulikottil Jacob
}

Received: November 15, 2016 / Published online: February 10, 2017

(C) The Author(s) 2017. This article is published with open access at Springerlink.com

\begin{abstract}
Introduction: Neovascular age-related macular degeneration (nAMD) is a chronic eye condition that causes severe deterioration of vision and ultimately blindness. Two vascular endothelial growth factor inhibitors are approved for nAMD treatment in Europe: ranibizumab and aflibercept. The European license for ranibizumab was updated with an individualized "treat and extend" (T\&E) regimen, which involves more proactive treatment based on changes in best corrected visual acuity (BCVA) and/or anatomical outcomes. The aim of this publication is to
\end{abstract}

Enhanced content To view enhanced content for this article go to http://www.medengine.com/Redeem/ F587F06006221056.

Electronic supplementary material The online version of this article (doi:10.1007/s12325-017-0484-0) contains supplementary material, which is available to authorized users.

A. Danyliv $(\bowtie)$

Novartis Ireland Limited, Dublin, Ireland

e-mail: andrii.danyliv@novartis.com

J. Glanville · R. McCool

York Health Economics Consortium, University of

York, York, UK

A. Ferreira · A. Skelly

Novartis Pharma AG, Basel, Switzerland

R. P. Jacob

Novartis Pharmaceuticals UK Limited, Surrey, UK compare the efficacy of the ranibizumab $T \& E$ regimen with other approved dosing regimens for nAMD on the basis of outcomes identified from a systematic review and subsequent NMA. Methods: Following a systematic search of publications, to identify relevant studies, a repeated-measures network meta-analysis (NMA) was performed to estimate the relative effectiveness of ranibizumab T\&E versus approved dosing regimens of ranibizumab and aflibercept. The analysis focused on licensed treatment regimens for nAMD. We examined mean change from baseline in BCVA on the Early Treatment Diabetic Retinopathy Study (ETDRS) chart.

Results: The systematic literature review identified 22,949 records, of which 23 studies were included in the NMA. At 12 months, the ranibizumab T\&E dosing regimen vs ranibizumab pro re nata (PRN) was associated with small differences in change in BCVA, between 1.86 letter gain at 12 months and 2.35 letter gain at 24 months. A similar difference was observed in the aflibercept dosing regimen versus ranibizumab T\&E ; 1.94 letter gain at 12 months and 3.31 letter gain at 24 months. All doses of ranibizumab and aflibercept showed similar effectiveness, and the differences between treatment options were not significant.

Conclusion: This study used novel repeated-measures NMA to synthesize efficacy results when treatment effects were reported at multiple follow-up times. This 
repeated-measures NMA suggests that treating patients with the ranibizumab T\&E regimen yields similar effectiveness compared to other approved ranibizumab and aflibercept dosing regimens for nAMD treatment.

Funding: Novartis Pharmaceuticals UK Ltd, Surrey, UK.

Keywords: Aflibercept; Neovascular age-related macular degeneration; Network meta-analysis; Ophthalmology; Ranibizumab; Systematic review; Treat and extend; Vascular endothelial growth factor inhibitors

\section{INTRODUCTION}

Neovascular age-related macular degeneration (nAMD) is a leading cause of vision loss globally $[1,2]$. Studies have estimated that $8.7 \%$ of the worldwide population has AMD; the projected number of people worldwide with the disease is 196 million in 2020, which is projected to increase to 288 million by 2040 [3]. In 2013, over 338,000 individuals in the UK were affected by nAMD, with 50,000 cases resulting in blindness [4-6].

There have been significant advances in the management of nAMD since the approval of anti-VEGF therapies, and patients now have effective treatment options that can limit the progression to blindness [7-9]. Anti-VEGF injections aim to inhibit the growth of new, abnormal blood vessels. Two anti-VEGF therapies that target VEGF-A have been approved for intraocular use in nAMD cases by the European Medicines Association (EMA) for the treatment of nAMD [10, 11]: ranibizumab (Lucentis ${ }^{\circledR}$; Novartis Pharma AG, Basel, Switzerland) [10] and aflibercept (Eylea ${ }^{\circledR}$; Bayer Pharma AG, Berlin, Germany) [11].

With two approved anti-VEGF treatments and multiple dosing regimens reported in the UK clinical practice, decisions on the optimal treatment regimen are increasingly difficult. In September 2014, the license for ranibizumab was updated to include a new "treat and extend" (T\&E) regimen, in which patients are treated monthly until maximum visual acuity
(BCVA) is achieved and/or there are no signs of disease activity, and then treatment intervals are extended in a stepwise manner of no more than 2 weeks at a time until signs of disease activity or visual impairment recur [10]. The effectiveness of treating nAMD patients with ranibizumab according to the T\&E regimen has been demonstrated in two randomized controlled trials (RCTs) [12-14]. However, the main comparisons have been to unlicensed treatment and not to other approved dosing regimens. Thus indirect comparisons are needed to evaluate the relative effects of different treatments over time and to inform decision-makers on the optimal treatment choice [15-17].

Network meta-analysis (NMA) takes into account both direct and indirect evidence. In addition to the complexity created by changing dosing regimens, the trials that inform this comparison have varying lengths of follow-up and report the outcome of interest (mean change in BCVA) at different time intervals. In such a situation, it is important to utilize all available information for all the time points reported in the trials to be able to estimate accurately the relative treatment effectiveness. For these purposes, a repeated-measures NMA model was used to model the relative treatment effectiveness.

In clinical practice in the UK, both ranibizumab and aflibercept are recommended as first-line agents for the treatment of nAMD and the decision to use either agent is left to the treating physician [18]. The purpose of this publication is to compare the efficacy of the ranibizumab T\&E regimen with other approved dosing regimens for nAMD on the basis of outcomes identified from a systematic review and subsequent NMA.

\section{METHODS}

The NMA reported in this manuscript is an update of a review and NMA originally conducted in 2013 to compare the efficacy of a range of interventions for the treatment of nAMD. Results of the comparisons of ranibizumab T\&E versus approved dosing regimens of ranibizumab and aflibercept are presented in this manuscript. 


\section{Eligibility Criteria}

Trials were eligible for inclusion in the systematic review if they assessed adult patients with nAMD and all relevant synonyms (e.g., exudative macular degeneration, choroidal neovascularization, AMD, age-related macular degeneration (ARMD)). Trials of mixed populations were eligible if the results for patients with nAMD were reported separately. Prospective RCTs, which assessed BCVA and compared any of the following interventions, were eligible for inclusion in the NMA: ranibizumab, unlicensed bevacizumab, aflibercept, photodynamic therapy, placebo, sham, or best supportive care.

\section{Search Strategy}

For the update to the systematic review, we searched 18 databases, websites, trial registries and three conference websites between February and March 2016. The search strategy for MEDLINE is shown in Supplemental Fig. 1 (Fig. S1) and the other searches are available on request. This search strategy was originally developed in 2013, and then updated for this analysis taking into account relevant recent changes in indexing $[19,20]$. The searches were not limited by date, language, or document type. The information sources searched are shown in Supplemental Table 1 (Table S1). Search results were downloaded into Endnote and de-duplicated against each other and against the results of the original review.

\section{Study Selection}

The records were screened for relevance to the NMA on the basis of information in the title and abstract, by two independent reviewers. Any disagreements were discussed with a third reviewer. Full-text documents of all potentially relevant records were obtained and evaluated in detail against the eligibility criteria for participants, interventions, comparators, outcomes, and study design. Studies that were excluded following full-text review are reported in Appendix 1 along with a reason for exclusion.

\section{Data Extraction and Quality Assessment}

Data extraction and quality assessment were carried out by two reviewers independently with discrepancies resolved by a third reviewer. Data were extracted from all relevant trials in relation to the trial design and methodology, patient characteristics, details of the intervention and treatment regimens, and data on change in BCVA. Data were extracted at all time points reported for each trial. Study risk of bias was assessed according to the NICE STA guidance for assessing the quality of RCTs [21].

\section{Feasibility Assessment}

NMA guidelines require studies in the network to be sufficiently similar to facilitate a reliable comparison. We used the guidance produced by the Australian Pharmaceutical Benefits Advisory Committee (PBAC) [22] on best practice for the conduct of indirect and mixed treatment comparisons to guide this assessment. The feasibility assessment evaluated the similarity of the trials in relation to the methods employed in the trials, participant populations, similarity of treatments, and similarity of outcome measures.

\section{Network Meta-Analysis}

A list of unique dosing regimens was generated describing each of the treatments identified in the literature review (Table S7) and a network was constructed. Dakin et al. and Ding and Fu NMA models were used to analyze the data at all time points $[23,24]$ up to months 12 and 24 . This NMA framework allows for repeated measurements of a continuous endpoint showing random and systematic variation (without specifying any particular relationship between treatment effects and time) [23]. The model by Ding and $\mathrm{Fu}$ assumes that the relationship of the mean relative treatment effect with time follows a parametric model [24]. Both fixed and random effects models were fitted to the 1-24 months network using WinBugs. The model with the minimum deviance information criterion (DIC) was chosen. 


\section{Compliance with Ethics Guidelines}

This article is based on previously conducted studies and does not involve any new studies of human or animal subjects performed by any of the authors.

\section{RESULTS}

\section{Identification and Selection of Studies}

The searches identified 22,949 records (Fig. 1). After de-duplication against each other and against the search results of the original review $[19,20], 5587$ records were assessed for relevance. Thirty-four documents, reporting data for 14 trials, were identified from the update search as eligible for inclusion in the NMA. Twenty-one of these documents reported data for six trials, which had already been identified in the previous NMA [19]. For this analysis, the remaining 13 documents reported data for eight new trials to be considered for inclusion, of which four studies ultimately contributed to the comparisons of interest to this network (Table S2). A total of 25 trials ( 21 from the original review and two from the update search) were considered in the feasibility assessment. A preferred reporting items for systematic reviews and meta-analyses (PRISMA) flow diagram showing the study selection process is presented in Fig. 1.

\section{Network Development}

Feasible networks were identified for analyzing mean changes in BCVA, which differed depending on the time point. A number of potential sources of heterogeneity were identified in the feasibility assessment of the 25 candidate trials: in particular, the potential for heterogeneity due to differences in the methods used, differences in the interventions used (primarily with respect to the criteria used for assessing retreatment), and differences in the timing of reporting. However, following discussions with clinicians, it was decided that these differences would be acknowledged but all the studies would remain in the network.
Following an assessment of the available data, two trials, DETAIL [25] and Moon et al. [26], provided insufficient data to be included in the final analysis. Therefore, data from 23 trials were included in the NMA (Fig. S2, Tables S3-S6).

\section{Results of the NMA}

\section{Treatment Differences at 12 and 24 Months}

The random effects model fit the data better than the fixed effects model. The functional form assumed by Ding and Fu [24] to describe the time component in the treatment effects fit the data well, and better than the models described by Dakin et al. [23]. The frequency of treatment varied over time and therefore different networks were applied depending on the time point. All of the 23 candidate trials reported data on BCVA at one time point, but the frequency and duration varied across the trials (Table S3). Table S7 presents the assumptions regarding the equivalence of treatment regimens at $0-12$ months. After 12 months all the treatment regimens in the analysis were different, and hence no assumptions were necessary for the 24 month analysis.

In general, ranibizumab T\&E resulted in similar changes in BCVA compared to other treatment regimens. Compared to aflibercept, ranibizumab T\&E was associated with an incremental letter gain from baseline of 1.94 [95\% credible interval (CrI) -7.52 to 3.52] at 12 months and 3.31 (95\% CrI -9.23 to 2.66 ) letter gain at 24 months. The relative treatment effectiveness of ranibizumab T\&E was 1.86 (95\% CrI -7.38 to 3.61 ) letter gain at 12 months and 2.35 (95\% CrI -7.82 to 3.19) letter gain at 24 months (Table 1) compared to ranibizumab $0.5 \mathrm{mg} 3 \times$ monthly, then as needed. A forest plot comparing ranibizumab $\mathrm{T} \& \mathrm{E}$ with other approved treatment strategies is shown in Fig. 2.

\section{DISCUSSION}

There is limited availability of head-to-head randomized trial data on the comparative 


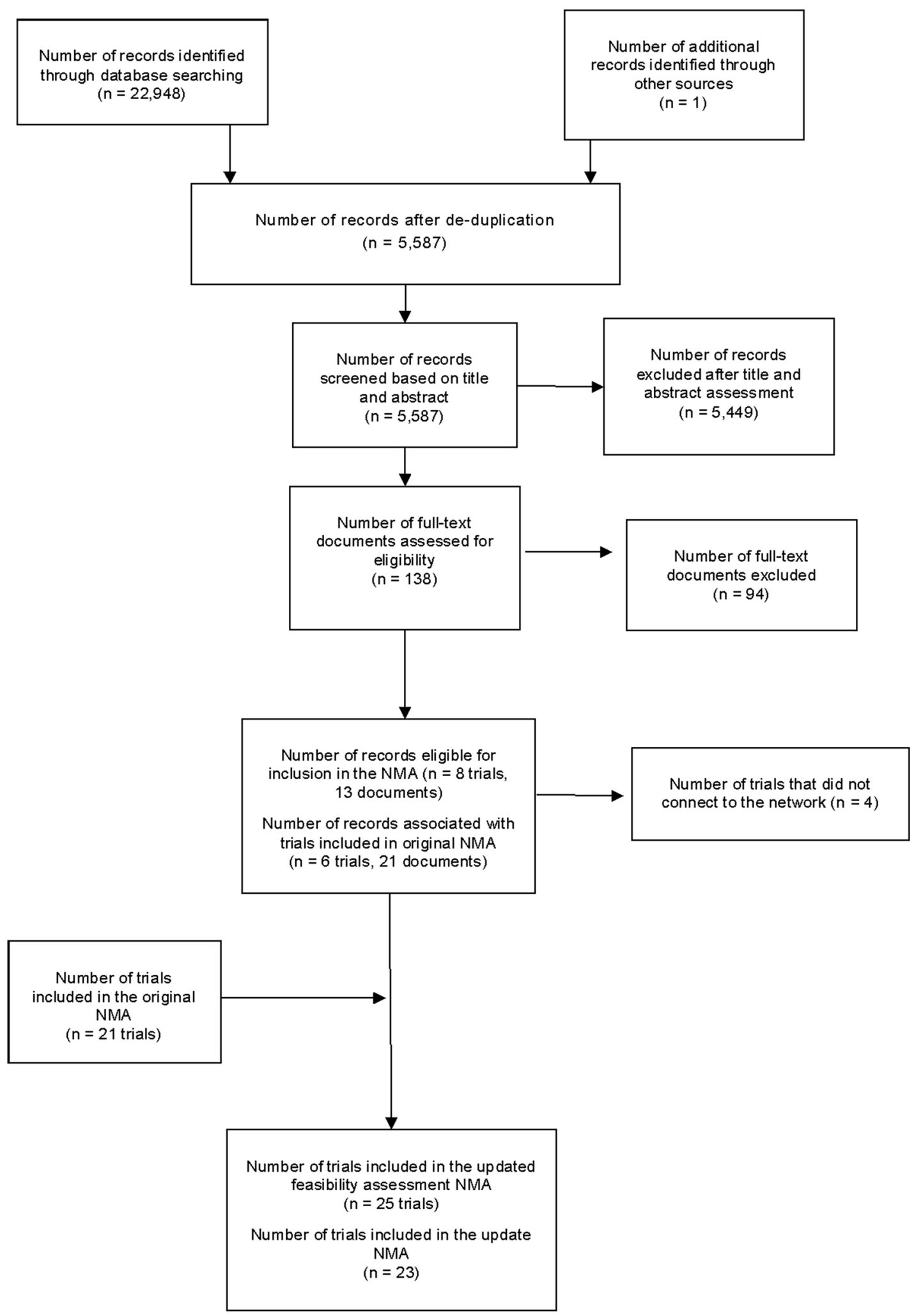

Fig. 1 Record selection process 
efficacy of different treatment regimens of ranibizumab for the treatment of nAMD; this analysis summarizes the available RCT evidence for the effectiveness of the ranibizumab T\&E regimen. The results of the analysis show ranibizumab T\&E to be an effective treatment regimen for nAMD. Ranibizumab T\&E showed minor increase in change in BCVA at 12 and 24 months compared to other licensed treatments.

These estimates are based on evidence from only two T\&E RCTs, of which only one informed the primary comparisons, and are subject to a considerable degree of uncertainty due to few data points. It is possible that the observed outcome may change if estimates from ranibizumab T\&E were based on a larger number of clinical trials. Until additional evidence is generated, this analysis provides insight on the treatment effects of ranibizumab T\&E in comparison to other approved treatment regimens for healthcare decision-makers, as ranibizumab T\&E is increasingly implemented in UK clinical practice. Furthermore, these results may be relevant in settings outside of the UK where the same approved anti-VEGF therapies are used in the clinic.

The principal strength of this study is that it accounts for the time structure in the data while comparing treatments, thus increasing the precision of the treatment estimates. Traditionally, the meta-analysis of trials with repeated measures was based on separate meta-analysis at each relevant time point, or analysis at the final time point of each trial. Such analysis would have not utilized all the available trial data from the different dosing regimens of interest at different time points; therefore, the estimates of the relative treatment effectiveness would have been less accurate.

Recently, several models for NMA of repeated measures have been presented [24]. The

Table 1 Differences in the change in visual acuity at 12 and 24 months between the treatments of interest

\begin{tabular}{|c|c|c|}
\hline & $\begin{array}{l}\text { Mean } \mid \text { median }[95 \% \mathrm{Crl}] \\
(p \text { value })\end{array}$ & $\begin{array}{l}\text { Mean } \mid \text { median }[95 \% \mathrm{Cr} l] \\
(p \text { value })\end{array}$ \\
\hline \multicolumn{3}{|l|}{12 months } \\
\hline & $\begin{array}{l}\text { Aflibercept } 2.0 \mathrm{mg} 6 \times 8 \text { weekly, } \\
\text { then as needed }\end{array}$ & $\begin{array}{l}\text { Ranibizumab } 0.5 \mathrm{mg} \\
3 \times \text { monthly, then as needed }\end{array}$ \\
\hline $\begin{array}{l}\text { Ranibizumab } 0.5 \mathrm{mg} 3 \times \text { monthly, } \\
\text { then as needed }\end{array}$ & $\begin{array}{l}-0.09 \mid-0.10 \\
{[-2.75 \text { to } 2.57]} \\
(0.47)\end{array}$ & \\
\hline $\begin{array}{l}\text { Ranibizumab } 0.5 \mathrm{mg} \text { treat } \\
\text { and extend }\end{array}$ & $\begin{array}{l}-1.95 \mid-1.94 \\
{[-7.52 \text { to } 3.52]} \\
(0.24)\end{array}$ & $\begin{array}{l}-1.86 \mid-1.86 \\
{[-7.38 \text { to } 3.61]} \\
(0.25)\end{array}$ \\
\hline \multicolumn{3}{|l|}{24 months } \\
\hline $\begin{array}{l}\text { Ranibizumab } 0.5 \mathrm{mg} 3 \times \text { monthly, } \\
\text { then as needed }\end{array}$ & $\begin{array}{l}-0.97 \mid-0.96 \\
{[-4.41 \text { to } 2.44]} \\
(0.28)\end{array}$ & \\
\hline $\begin{array}{l}\text { Ranibizumab } 0.5 \mathrm{mg} \text { treat } \\
\text { and extend }\end{array}$ & $\begin{array}{l}-3.32 \mid-3.31 \\
{[-9.23 \text { to } 2.66]} \\
(0.13)\end{array}$ & $\begin{array}{l}-2.35 \mid-2.35 \\
{[-7.82 \text { to } 3.19]} \\
(0.19)\end{array}$ \\
\hline
\end{tabular}



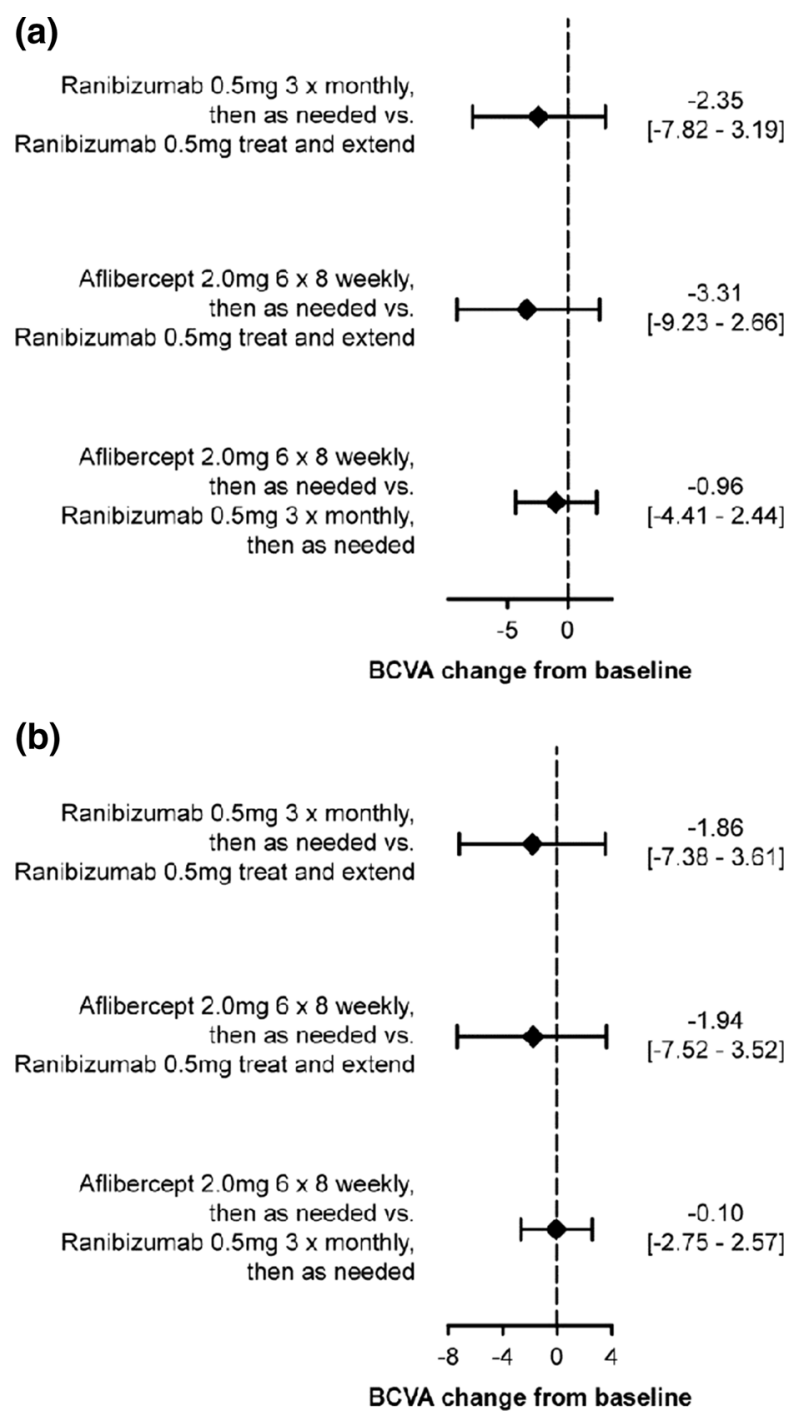

Fig. 2 Forest plots present the median treatment difference and 95\% CrIs of rainbizumab T\&E versus other approved treatment regimens at $\mathbf{a} 12$ months and $\mathbf{b}$ at 24 months

NMA was undertaken using the most robust methodology for NMA of repeated measures, providing accurate estimates of the effects of ranibizumab T\&E in the absence of head-tohead RCT data. Moreover, the Ding and Fu model proposes a parametric model for the response time of each treatment and can be used to extrapolate the data at unobserved time points. Therefore, NMA of repeated measures should be explored more systematically in appropriate future nAMD studies, as it allows for an evaluation of the treatment effects over time and overcomes some of the limitations of standard NMA. The concept of a single full network, as used in standard NMA, can be somewhat misleading, as the full network changes over time reflecting changes in individual dosages. In such a situation, using NMA of repeated measures could offer a greater advantage in terms of obtaining more precise estimates at specific time points.

NMA is subject to the same limitations as pairwise meta-analysis [27], mainly related to the quality of the individual studies and publication biases. Assessments of the risk of bias of the included trials showed there to be significant variability in the quality of the included studies, with a number of the studies included in our analysis failing to take appropriate steps to minimize bias. While it is difficult to assess formally the impact of including these within the context of the NMA analysis, there is the potential that the estimates generated from our analysis are subject to bias as a result of the inclusion of these studies.

As described above, our analysis is based on an extensive search of the literature. Although we identified several studies, the large number of dosing regimens in the network means that the network analyzed is relatively sparse, giving the estimates considerable uncertainty. Another limitation of the current analysis is that observable heterogeneity based on baseline BCVA has not been addressed. It has been demonstrated in previous studies that a significant association exists between baseline BCVA and subsequent changes in BCVA following treatment [28]. It was therefore proposed within the current analysis to utilize meta-regression techniques to adjust estimates for baseline BCVA. Our study was only able to use aggregate information on baseline BCVA, because individual patient data from each of the trials were unavailable. Actual treatment effectiveness may therefore vary according to a patient's BCVA in a way that we were not able to demonstrate in this analysis. NMA of repeated measures provides a clear advantage over standard NMA, when the outcomes of interest across different trials are not reported at the same time points. Despite these limitations, our results show that 
ranibizumab T\&E is similarly effective to other approved treatment regimens of nAMD.

\section{CONCLUSION}

This study has shown the comparative effectiveness of ranibizumab T\&E with other approved treatment regimens for nAMD. NMA of repeated measures provides a clear advantage over standard NMA, when the outcomes of interest across different trials are not reported at the same time points.

\section{ACKNOWLEDGEMENTS}

This study was funded by Novartis Pharmaceuticals UK Ltd, Surrey, UK.

The original analysis was undertaken by Kelly Fleetwood at Quantics Consulting Ltd and was funded by Novartis.

The authors were assisted in the preparation of the manuscript by Jackie L. Johnson, Novartis Ireland Limited, Dublin, Ireland. Writing support was funded by the study sponsor. Novartis funded the publication charges and open access fee.

All named authors meet the International Committee of Medical Journal Editors (ICMJE) criteria for authorship for this manuscript, take responsibility for the integrity of the work as a whole, and have given final approval to the version to be published.

Disclosures. Alberto Ferreira is an employee of Novartis Pharma AG and owns stock in Novartis. Adrian Skelly is an employee of Novartis Pharma AG and owns stock in Novartis. Ruth Pulikottil Jacob is an employee of Novartis Pharmaceuticals UK Ltd. Andriy Danyliv is employed by Novartis Ireland. Julie Glanville is an employee of YHEC. Rachael $\mathrm{McC}$ Cool is an employee of YHEC.

Compliance with Ethics Guidelines. This article is based on previously conducted studies and does not involve any new studies of human or animal subjects performed by any of the authors.
Data Availability. All data generated or analyzed during this study are included in this published article/as supplementary information files.

Open Access. This article is distributed under the terms of the Creative Commons Attribution-NonCommercial 4.0 International License (http://creativecommons.org/licenses/ by-nc/4.0/), which permits any noncommercial use, distribution, and reproduction in any medium, provided you give appropriate credit to the original author(s) and the source, provide a link to the Creative Commons license, and indicate if changes were made.

\section{REFERENCES}

1. Congdon N, O'Colmain B, Klaver CC, et al. Causes and prevalence of visual impairment among adults in the United States. Arch Ophthalmol. 2004;122(4):477-85.

2. Pascolini D, Mariotti SP, Pokharel GP, et al. 2002 Global update of available data on visual impairment: a compilation of population-based prevalence studies. Ophthalmic Epidemiol. 2004;11(2):67-115.

3. Wong WL, Su X, Li X, et al. Global prevalence of age-related macular degeneration and disease burden projection for 2020 and 2040: a systematic review and meta-analysis. Lancet Glob Health. 2014;2(2):e106-16.

4. Bunce C, Xing W, Wormald R. Causes of blind and partial sight certifications in England and Wales: April 2007-March 2008. Eye. 2010;24(11):1692-9.

5. Menon G, Walters G. New paradigms in the treatment of wet AMD: the impact of anti-VEGF therapy. Eye. 2009;23(Suppl 1):S1-7.

6. Owen CG, Jarrar Z, Wormald R, Cook DG, Fletcher AE, Rudnicka AR. The estimated prevalence and incidence of late stage age related macular degeneration in the UK. $\mathrm{Br} \mathrm{J}$ Ophthalmol. 2012;96(5):752-6.

7. Wong TY, Liew G, Mitchell P. Clinical update: new treatments for age-related macular degeneration. Lancet. 2007;370(9583):204-6.

8. Bressler NM, Doan QV, Varma R, et al. Estimated cases of legal blindness and visual impairment 
avoided using ranibizumab for choroidal neovascularization: non-Hispanic white population in the United States with age-related macular degeneration. Arch Ophthalmol. 2011;129(6):709-17.

9. Lim LS, Mitchell P, Seddon JM, Holz FG, Wong TY. Age-related macular degeneration. Lancet. 2012;379(9827):1728-38.

10. European Medicines Agency (EMA). Lucentis $10 \mathrm{mg} / \mathrm{ml}$ solution for injection. Summary of product characteristics. http://www.ema.europa.eu/ docs/en_GB/document_library/EPAR_-_Product_ Information/human/000715/WC500043546.pdf. Accessed 11 Feb 2016. 2009.

11. European Medicines Agency (EMA). Eylea $40 \mathrm{mg} / \mathrm{ml}$ solution for injection in pre-filled syringe. Summary of product characteristics. http://www.ema.europa. eu/docs/en_GB/document_library/EPAR_-_Product_ Information/human/002392/WC500135815.pdf. Accessed Feb 2016. 2012.

12. Berg K, Hadzalic E, Gjertsen I, et al. Ranibizumab or bevacizumab for neovascular age-related macular degeneration according to the lucentis compared to avastin study treat-and-extend protocol: two-year results. Ophthalmology. 2016;123(1):51-9.

13. Berg K, Pedersen TR, Sandvik L, Bragadottir R. Comparison of ranibizumab and bevacizumab for neovascular age-related macular degeneration according to LUCAS treat-and-extend protocol. Ophthalmology. 2015;122(1):146-52.

14. Wykoff CC, Croft DE, Brown DM, et al. Prospective trial of treat-and-extend versus monthly dosing for neovascular age-related macular degeneration: TREX-AMD 1-year results. Ophthalmology. 2015;122(12):2514-22.

15. Caldwell DM, Ades AE, Higgins JP. Simultaneous comparison of multiple treatments: combining direct and indirect evidence. BMJ. 2005;331(7521):897-900.

16. Jansen JP, Fleurence R, Devine B, et al. Interpreting indirect treatment comparisons and network meta-analysis for health-care decision making: report of the ISPOR task force on indirect treatment comparisons good research practices: part 1 . Value Health. 2011;14(4):417-28.

17. Lu G, Ades AE. Combination of direct and indirect evidence in mixed treatment comparisons. Stat Med. 2004;23(20):3105-24.

18. Hykin P, Chakravarthy U, Lotery A, McKibbin M, Napier J, Sivaprasad S. A retrospective study of the real-life utilization and effectiveness of ranibizumab therapy for neovascular age-related macular degeneration in the UK. Clin Ophthalmol. 2016;10:87-96.

19. Hodgson R, Barata T, Fleetwood K, Edwards M, Glanville J. Indirect treatment comparison of lucentis for wet age related macular degeneration. York Health Economics Consortium, York. 2014

20. Fitzgerald A, Cikalo M, Sreekanta A, et al. Systematic review of the clinical effectiveness and safety of lucentis for wet age-related macular degeneration. York: York Health Economics Consortium; 2014

21. National Insititute for Health and Care Excellence. Final guidance on the use of ranibizumab and pegaptanib for the treatment of age-related macular degeneration (AMD). London: National Insititute for Health and Care Excellence; 2008 (press release).

22. Australian Pharmaceutical Benefits Advisory Committee Indirect Comparisons Working Group. Report of the Indirect Comparisons Working Group to the Pharmaceutical Benefits Advisory Committee: Assessing Indirect Comparisons. Canberra: Australian Department of Health and Ageing, Pharmaceutical Benefits Advisory Committee, 2009.

23. Dakin HA, Welton NJ, Ades AE, Collins S, Orme M, Kelly S. Mixed treatment comparison of repeated measurements of a continuous endpoint: an example using topical treatments for primary open-angle glaucoma and ocular hypertension. Stat Med. 2011;30(20):2511-35.

24. Ding $\mathrm{Y}, \mathrm{Fu} \mathrm{H}$. Bayesian indirect and mixed treatment comparisons across longitudinal time points. Stat Med. 2013;32(15):2613-28.

25. London NJ, Fung AE, Dugel PU, et al. A Prospective evaluation of ranibizumab for retinal pigment epithelial detachments in neovascular age-related macular degeneration. Invest Ophthalmol Vis Sci. 2009;50(5):2379.

26. Moon E, et al. Monthly versus PRN ranibizumab for neovascular AMD: a randomized phase 2 open-label study. Invest Ophthalmol Vis Sci 2011;52(6):133.

27. Cameron C, Fireman B, Hutton B, et al. Network meta-analysis incorporating randomized controlled trials and non-randomized comparative cohort studies for assessing the safety and effectiveness of medical treatments: challenges and opportunities. Syst Rev. 2015;4:147.

28. Busbee BG, Ho AC, Brown DM, et al. Twelve-month efficacy and safety of $0.5 \mathrm{mg}$ or $2.0 \mathrm{mg}$ ranibizumab in patients with subfoveal neovascular age-related macular degeneration. Ophthalmology. 2013;120(5):1046-56. 